\title{
Resource Leveling in Construction Projects with Activity Splitting and Resource Constraints: A Simulated Annealing Optimization
}

\begin{tabular}{|c|c|}
\hline Journal: & Canadian Journal of Civil Engineering \\
\hline Manuscript ID & cjce-2017-0670.R1 \\
\hline Manuscript Type: & Article \\
\hline $\begin{array}{r}\text { Date Submitted by the } \\
\text { Author: }\end{array}$ & 01-Jun-2018 \\
\hline Complete List of Authors: & $\begin{array}{l}\text { Piryonesi, S. Madeh; University of Toronto, Civil Engineering; } \\
\text { Nasseri, Mehran; Sharif University of Technology, Industrial } \\
\text { Engineerin } \\
\text { Ramezani, Abdollah; University of Mazandaran }\end{array}$ \\
\hline $\begin{array}{r}\text { Is the invited manuscript } \\
\text { for consideration in a } \\
\text { Special Issue? : }\end{array}$ & Not applicable (regular submission) \\
\hline Keyword: & $\begin{array}{l}\text { Resource management, Resource leveling, Simulated annealing, } \\
\text { Meta-heuristic, Activity splitting }\end{array}$ \\
\hline
\end{tabular}




\title{
1 Resource Leveling in Construction Projects with Activity Splitting
}

\section{and Resource Constraints: A Simulated Annealing Optimization}

\author{
S. Madeh Piryonesi ${ }^{1}$, Mehran Nasseri ${ }^{2}$, Abdollah Ramezani ${ }^{3}$
}

Overtime and over-budget construction projects are not pleasant to any stakeholder. Stakeholders want construction projects to be completed without delay and excessive cost. It is possible to meet these objectives by using resource management techniques such as resource leveling. Due to the limitation of resources and different types of them in a construction project, optimizing the resource utilization is crucial. In this paper, a meta-heuristic simulated annealing resource leveling model is presented. The novelty of this model lies not only in the type of modeling and optimization but also in its assumptions. Our model simultaneously allows activities to split and considers a limitation in resource availabilities. The developed model was implemented in a computer program. Then, it was applied to an example from the literature of resource leveling. The model successfully solved the problem. The results of our model are compared with those already available in the literature.

\section{Introduction}

21 A common definition for project is a temporary endeavor undertaken to create a unit product, service or result (PMI

22 2007). Based upon a more extended definition, a project is a unique and temporary endeavor that can be subdivided

23 into various activities that require time and renewable resources, such as machines, equipment, or manpower, for their

24 execution. Therefore, projects share the quality of uniqueness. Construction projects are no exception. They are also

\footnotetext{
${ }^{1}$ Ph.D. Candidate, University of Toronto, School of Civil Engineering. Email: madeh.piryonesi@mail.utoronto.ca

${ }^{2}$ Graduate Student, Sharif University of Technology, Department of Industrial Engineering

${ }^{3}$ Assistant Professor, Mazandaran University of Science and Technology, Babol, Iran
} 
1 unique and temporary, hence more difficult to manage. Furthermore, a construction project often involves general temporal constraints among activities resulting from technological or organizational restrictions. Thus, developing a project schedule is necessary for managing such constraints and restrictions. Project scheduling consists of determining start times for all activities such that temporal and/or resource constraints are satisfied, and a series of objective are optimized (Hendrickson and Au 2003; PMI 2007).

Construction resources are expensive, and their fluctuation imposes unwanted costs on contractors. To avoid these undesirable fluctuations, and their consecutive extra costs, contractors usually use resource leveling. According to (PMI 2007), resource leveling is defined as a technique in which the start and finish time of activities are adjusted

10 based on resource constraints in the hope that the demand for resources would be balanced with the available supply.

11 Resource leveling aims at minimizing the fluctuation in resource utilization throughout the project.

13 As a particular type of project, the construction projects are heavily dependent on the resource leveling (Hendrickson

14 and Au 2003). Construction schedules generated by network scheduling techniques often need to be modified to reduce

15 significant fluctuations in resource utilization. As mentioned, these fluctuations impose negative effects on different 16 stakeholders specially the contractor. Some of these effects are the cost of hiring and releasing workers in a short 17 interval, the disruption in the learning curve effects and maintaining an unproductive level of workforce on site that 18 keeps some workers idle during low demand periods (El-Rayes and Jun 2009). Consequently, over the last few 19 decades, researchers have tackled the resource leveling problems (RLPs) in the construction projects (Easa 1989; Leu 20 et al. 2000; El-Rayes and Jun 2009; Koulinas and Anagnostopoulos 2012; Menesi and Hegazy 2015).

22 Different methods have been applied to RLPs, specially optimization models, which have been widely used in 23 construction engineering and management (Leu et al. 2000; El-Rayes and Jun 2009; Kaveh et al. 2013; Piryonesi 24 and Tavakolan 2017; Moradi et al. 2017). These methods could be categorized in three major groups: analytical or 25 mathematical methods, heuristic methods and meta-heuristic methods (Leu et al. 2000). As an example of 26 mathematical programing, Easa (1989) used an integer programming to solve the RLP in construction projects. As 27 another example of mathematical programming, Rieck et al. (2012) applied a mixed-integer linear programming to 28 the problem. Similar studies are available in the literature. Although the analytical methods are completely accurate 
1 in finding optimal solutions, but they need an extensive computational effort for large problems, hence sometimes

2 impractical. This issue is especially important when tackling resource leveling problems. (Easa 1989; Harris 1990;

3 Piryonesi and Tavakolan 2017; Najibi and Devineni 2018).

5 Resource leveling problems (RLPs) are among the problems with combinatorial explosion. Therefore, using analytical

6 methods to solve RLPs for projects with many tasks is not reasonable. Heuristic methods have been used as a simple

7 and efficient tool to avoid this pitfall (Easa 1989). Two famous examples of heuristic methods are PACK (Harris

8 1990) and NASTRAT (Padilla and Carr 1991). Other heuristic rules to solve RLPs are available in the literature (Wiest

9 and Levy 1977; Antill and Woodhead 1990). Notwithstanding their efficiency, such heuristic models have a significant

10 drawback. They are problem-dependent, hence cannot be equally applied to different projects.

12 The limitations of mathematical and heuristic methods prompted researchers to use more powerful techniques such as

13 meta-heuristic algorithms. Genetic algorithms (GAs) are among the most powerful and popular meta-heuristic

14 algorithms. Many researchers have successfully solved RLPs using GAs (Leu et al. 2000; Leu and Hung 2002; Toklu

15 2002; Kim 2009; El-Rayes and Jun 2009; Zahraie and Tavakolan 2009). Leu et al. (2000) used the GA to solve the

16 problem of resource leveling in construction projects. Their GA did not allow activity splitting. They developed a

17 prototype of a decision support system (DSS) for analyzing different what-if scenarios. Another example of using

18 GAs is done by Leu and Hung (2002). They considered the duration of activities to be probabilistic and used Monte

19 Carlo simulation to handle probabilities. Their model did not allow the activities to split either. Toklu (2002) solved

20 the problem of resource leveling using GAs with and without resource constraints. Scheduling project without resource

21 constraint, however, could be done using methods such as the CPM.

23 The studies mentioned above were dealing with one type of resource and did not allow activity splitting. Other studies

24 on solving RLPs with multiple resources are available as well. Damci et al. (2013) used a GA to solve the multi-

25 resource leveling problem in a line of balance scheduling. Another example of using GAs in solving multiple resources

26 RLPs in construction is available at Ponz-Tienda et al. (2013). Using a Weibull distribution to establish an estimation

27 of the global optimum as a termination condition made the last work different from other previously developed GAs. 
1 In real construction projects, activities could be, and sometimes even must be, split. Splitting the activities in resource

2 leveling could result in a smoother profile of resources. The studies mentioned above did not allow the tasks to split.

3 However, research has been done on the impact of splitting activities on resource leveling. For instance, Peteghem

4 and Vanhoucke (2010) reported that splitting activities resulted in smaller fluctuation of resources. They used a genetic

5 algorithm to solve the problem of multi-mode resource leveling. Another example of allowing splitting is done by

6 Hashemi Doulabi et al. (2011) who used a hybrid GA to solve an RLP.

8 Other meta-heuristic algorithms have been used as well. Geng et al. (2011) deployed an ant colony optimization

9 algorithm to tackle nonlinear RLPs. Another example is a tabu search-based algorithm that was proposed by Koulinas

10 and Anagnostopoulos (2013) for solving construction RLPs with limited resource availabilities. Simulated annealing

11 (SA) is another algorithm used by researchers, such as Son and Skibniewski (1999) Anagnostopoulos and Koulinas

12 (2010), to solve RLPs. Bouleimen and Lecocq (2003) used a simulated annealing for the resource-constrained project

13 scheduling problem with multiple resources. One of the advantages of their study was proving the effectiveness of

14 choosing feasible initial solutions over random solutions. Their study, however, did not allow activity splitting. Based

15 on what was discussed above, there has been limited effort on solving RLPs with simulated annealing which is capable

16 of considering activity splitting and resource constraints. Developing such a model is the focus of this research.

18 As explained above, researchers have used various optimization algorithms in solving RLPs. After reviewing the

19 literature of resource leveling and studying the strengths and the weaknesses of previous models, a meta-heuristic

20 simulated annealing algorithm is applied to the RLP in construction projects. What makes this study different from

21 previous research is the type of modeling that makes both activity splitting and considering the limited resource

22 availabilities possible. As explained, limited research is done on integrating activity splitting into simulated annealing

23 optimization. In the real construction projects, sometimes an activity cannot be completed in a continuous time due to

24 resource limitations. Although this activity splitting might cause extra costs, but it will result in a smoother resource

25 profile (Hashemi Doulabi et al. 2011). 


\section{$1 \quad$ 2. Simulated Annealing}

2 Simulated annealing (SA) is a probabilistic meta-heuristic method proposed by Kirkpatrick et al. (1983) and Černý

3 (1985). As the name of algorithm suggests, it is imitating the process of physical annealing of a solid, in which a

4 crystalline solid is heated and then allowed to cool gradually until it achieves its most regular crystal configuration

5 (i.e. the lowest amount of energy). Some features such as ease of implementation, convergence properties and its use

6 of hill-climbing moves to escape local optima have made it a popular technique over the past decades. It is usually

7 used to solve discrete, and to a lesser extent, continuous optimization problems (Henderson et al. 2003).

8 The high-level algorithm of SAs could be described as follows.

10 Select an initial solution (current solution)

11 Select a temperature change counter

12 Select a cooling schedule

13 Select an initial temperature

14 Select a repetition schedule that defines the number of iterations executed at each temperature

15 Repeat

16 Generate a solution (candidate solution)

17 If the candidate solution was better than current solution:

18 Replace the current with the candidate

19 Otherwise replace it with a probability

20 Until stopping criteria are met

22 Note that this is just a rough description of how SA algorithm works. For a better understanding of the algorithm used 23 in this paper see Figures 1 and 2.

\section{3. Problem formulation}

26 Based on given explanations, a new model was developed for the problem of resource leveling. The common

27 mathematical formulation of resource leveling problem is used to define the objective function (Hegazy 1999).

28 Accordingly, the problem may be formulated as follows: 
12 where $n=$ the number of activities, $T=$ the feasible time of project that is usually the output of a CPM analysis, $r_{t}=$

13 the required resource in time $t, x_{i t}=$ a binary variable that takes a value 1 if activity $i$ is done at time $t$ and otherwise

14 takes $0, d_{i}=$ the duration of activity $i, s_{i t}=$ an integer number that is calculated by subtracting $\sum_{\mathrm{k}=1}^{\mathrm{t}} \mathrm{x}_{\mathrm{ik}}$ from $d_{i}$ and

15 varies from $d i$ (as activity $i$ is not started yet) to 0 (as activity $i$ is completed), $M=$ a very large number, $z_{i t}=$ a binary

16 variable to oblige the precedency of every activity $i$ to every activity $j, r_{i t}=$ resources that are available at time $t$ for

17 activity $i$ and $R_{t}=$ the total number of available resources at time $t$ in the project.

19 The objective is to minimize the difference between the resources needed for every activity in subsequent unit of time

20 during the project (i.e. rt and rt-1). Equation (1b) is used to set the duration of activities. The constraints (1c) to (1f)

21 handle the precedency of activities. For more convenience, the variable $s_{i t}$ is defined to incorporate the splitting of

22 activities. It is worth noting that $s_{i t}$ cannot be negative due to Equation (1b). The activity $j$ will not start until $z_{i t}$ would

23 take a value 1 that means the activity $i$ is completed ( $s_{i t}$ becomes 0 ). Another constraint that is addressed in this model

24 is the limitation in resources. Dropping the last constraint will result in an RLP with unlimited resources. 
1 The presented model is a mixed integer non-linear programming (MINP) model with $2 n T$ variables and $4 n+5 T$ 2 constraints. Obtaining an optimal solution (i.e. using analytical methods) for this model is difficult. Currently, only

3 problems with a very limited number of activities can be solved by software such as GAMS and Lingo. Motivated by

4 this situation, a meta-heuristic procedure is developed in this article, which uses a simulated annealing to tackle the 5 problem.

\section{4. Resource leveling using SA}

8 As it was explained above in section 2, solving a problem using SA needs initial solutions. These initial solutions are 9 taken as current solutions and then new solutions will be generated. The new solutions and the current ones are 10 compared, and the best are selected. Generating random solutions may be an approach for generating new solutions, 11 but it is not effective enough. The randomness in initial solutions creates several insufficiencies including slower rate 12 of annealing and larger number of iterations (Bouleimen and Lecocq 2003). Therefore, the answer of the CPM analysis 13 is taken as an initial solution, as it satisfies the precedence relations and will be certainly a feasible solution. To 14 increase the effectiveness of model, new solutions are generated by selecting two points randomly between the earliest 15 start (ES) and the latest finish (LF) of an activity and then swapping them. This process is illustrated in Figure 1. If 16 these new solutions violate the constraints of model, a very large value is assigned to their associated objective function 17 values. On the other hand, the feasible solutions are evaluated and will be selected, if they are better than the current 18 answer. Otherwise, they will be chosen with a probability. The flowchart shown in Figure 2 explains the steps of the 19 algorithm.

Insert Figure 2

\section{5. Application example}

25 In this section, an example is extracted from the literature and solved to show the effectiveness of model. The example 26 is an 11-activity CPM network from Harris (1990). This is a single resource leveling problem. The precedency of the 27 activities, their resource demands and their durations are shown in Figure 3. First, the network is analyzed by CPM, 
1 and the result is presented in Table 1. This analysis gives the duration of project without considering any limitation in 2 resources.

Insert Figure 3

\section{Insert Table 1}

8 It is worth pointing out that this example is identically solved by Harris (1990) and Leu et al. (2000). Therefore, to be

9 able to compare the results with the answers of previous studies, the objective function is reformulated in the following 10 format:

11

$12 \quad$ Minimize $Z=\sum_{t=1}^{T}\left[\left|\sum_{i=1}^{n} r_{i} x_{i t}-\sum_{i=1}^{n} \frac{\left(r_{i} d_{i}\right)}{T}\right|\right]$

13 This formulation was first used by Easa (1989), in which the deviation of the required resources from a uniform

14 desired resource usage is minimized. The minimum $Z$ for this problem reported by Leu et al. (2000) and Harris

15 (1990) was 25, while before leveling the value of $Z$ was 69.

16

17 The problem was solved based on the model presented in Equation (1) and using the proposed simulated annealing 18 algorithm described in section 4. The algorithm was implemented by MATLAB 2012b run on a personal computer 19 with a $2.66 \mathrm{GHz}$ Intel ${ }^{\circledR}$ and $4 \mathrm{~GB}$ physical memory. The parameters of the SA are shown in Table 2. The minimum $Z$ 20 found by the model is 22.125 which is clearly better than the answers of two aforementioned studies (i.e. (Leu et al. 21 2000) and (Harris 1990)). The superiority of this model was somehow predictable, as it has the splitting capability 22 that results in more flexibility in the problem and obtaining better answers.

\section{Insert Table 2}

26 The process of improving the objective function with more iterations and the convergence of the algorithm is shown

27 in Figure 4. Figure 4 demonstrates that the value of objective function experiences a very steep decrease in the first 
1 few iterations. Later, after 19 iterations the algorithm converges, and the curve of Figure 4 becomes completely flat.

2 It means that the algorithm has reached the optimal answer.

\section{Insert Figure 4}

6 The optimal answer is shown in a bar chart in Figure 5. Figure 5 shows that when an activity $i$ is done at time $t$, the

7 binary variable $x_{i t}$ takes a value 1 and otherwise 0 . Thus, the activities that are split comprise 0 digits between 1 digits.

8 Tasks $\mathrm{A}$ and $\mathrm{E}$ are examples of split activities. On the other side, the intact activities are shown by a number of sequential 1s, without 0s in between. Activities B and D are examples of non-split tasks in this example.

\section{Insert Figure 5}

13 The result of solved example proved that our model is effective in solving a typical RLP. The splitting capability

14 made our model more flexible, hence its resource fluctuation smaller. However, one might argue that splitting some

15 activities might result in an increase in cost. Therefore, the splitting should not be conducted randomly or arbitrarily.

16 This is a valid criticism. In real world, contractor must conduct a cost-benefit analysis before choosing a specific

17 resource leveling model. In other words, the trade-off between the extra cost of a non-smooth resource profile and the

18 cost of activity-splitting should not be overlooked. Cost analysis is not within the scope of this study. An example of

19 a study on cost optimization in resource leveling with splitting is conducted by Hariga and El-Sayegh (2010). A

20 practical solution for incorporating such trade-off in the presented model would be as follows. First, those activities

21 that are not supposed to split must be identified. Later, a penalty should be assigned to these activities. Following this

22 approach, the activities that are not supposed to split will remain intact.

\section{6. Conclusions and recommendations}

25 In this paper, a new MINP model was developed to formulate the single RLP. To overcome the drawbacks of 26 analytical and heuristic optimization methods a meta-heuristic simulated annealing was presented. The model was

27 implemented using MATLAB. We then tested the model by an example from the literature of resource leveling. Our 28 model found the optimal answer successfully. Since, in real-world construction projects, activities are sometimes split 
1 due to resource limitation, the splitting capability was incorporated in the model. Adding this capability resulted in a

2 more flexible model and better answers in comparison to previous studies, which did not allow splitting.

3

4 Splitting the activities was mentioned as an advantage of our model. However, arbitrary splitting could be a limitation

5 in some projects. Splitting may not be allowable for some particular activities. Therefore, in the course of future

6 research, a new model will be proposed to allow splitting only in a limited number of activities not all of them. In

7 addition, a new approach could be used to generate the neighborhoods to make the optimization algorithm more

8 efficient.

10 7. References

11 Anagnostopoulos, K.P., and Koulinas, G.K. 2010. A simulated annealing hyperheuristic for construction resource

12 levelling. Construction Management and Economics, 28(2): 163-175. Routledge .

13 doi:10.1080/01446190903369907.

14 Antill, J.M., and Woodhead, R.W. 1990. Critical path methods in construction practice. Wiley.

15 Bouleimen, K., and Lecocq, H. 2003. A new efficient simulated annealing algorithm for the resource-constrained

16 project scheduling problem and its multiple mode version. European Journal of Operational Research, 149(2):

17 268-281. North-Holland. doi:10.1016/S0377-2217(02)00761-0.

18 Černý, V. 1985. Thermodynamical approach to the traveling salesman problem: An efficient simulation algorithm.

19 Journal of Optimization Theory and Applications, 45(1): 41-51. Kluwer Academic Publishers-Plenum

$20 \quad$ Publishers. doi:10.1007/BF00940812.

21 Damci, A., Arditi, D., and Polat, G. 2013. Multiresource Leveling in Line-of-Balance Scheduling. Journal of

22 Construction Engineering and Management, 139(9): 1108-1116. doi:10.1061/(ASCE)CO.1943-

$23 \quad 7862.0000716$.

24 Easa, S.M. 1989. Resource Leveling in Construction by Optimization. Journal of Construction Engineering and

25 Management, 115(2): 302-316. doi:10.1061/(ASCE)0733-9364(1989)115:2(302).

26 El-Rayes, K., and Jun, D.H. 2009. Optimizing Resource Leveling in Construction Projects. Journal of Construction

27 Engineering and Management, 135(11): 1172-1180. doi:10.1061/(ASCE)CO.1943-7862.0000097.

28 Geng, J., Weng, L., and Liu, S. 2011. An improved ant colony optimization algorithm for nonlinear resource- 
leveling problems. Computers \& Mathematics with Applications, 61(8): 2300-2305. Pergamon. doi:10.1016/J.CAMWA.2010.09.058.

Hariga, M., and El-Sayegh, S.M. 2011. Cost Optimization Model for the Multiresource Leveling Problem with Allowed Activity Splitting. Journal of Construction Engineering and Management, 137(1): 56-64. doi:10.1061/(ASCE)CO.1943-7862.0000251.

Harris, R.B. 1990. Packing Method for Resource Leveling (Pack). Journal of Construction Engineering and Management, 116(2): 331-350. doi:10.1061/(ASCE)0733-9364(1990)116:2(331).

Hegazy, T. 1999. Optimization of Resource Allocation and Leveling Using Genetic Algorithms. Journal of Construction Engineering and Management, 125(3): 167-175. doi:10.1061/(ASCE)07339364(1999)125:3(167).

Henderson, D., Jacobson, S.H., and Johnson, A.W. 2003. The Theory and Practice of Simulated Annealing. In Handbook of Metaheuristics. Kluwer Academic Publishers, Boston. pp. 287-319. doi:10.1007/0-306-48056-

14 Hendrickson, C.T., and Au, T. 2003. Project management for construction : fundamental concepts for owners, 15 engineers, architects, and builders. : 537. Prentice Hall, Pittsburgh. Available from http://pmbook.ce.cmu.edu/ $16 \quad$ [accessed 15 September 2017].

17 Hossein Hashemi Doulabi, S., Seifi, A., and Shariat, S.Y. 2011. Efficient Hybrid Genetic Algorithm for Resource Leveling via Activity Splitting. Journal of Construction Engineering and Management, 137(2): 137-146. doi:10.1061/(ASCE)CO.1943-7862.0000261.

Kaveh, A., Motie Share, M.A., and Moslehi, M. 2013. Magnetic charged system search: a new meta-heuristic algorithm for optimization. Acta Mechanica, 224(1): 85-107. Springer Vienna. doi:10.1007/s00707-012-0745-

25 Kirkpatrick, S., Gelatt, C.D., and Vecchi, M.P. 1983. Optimization by simulated annealing. Science (New York, 6.

Kim, J.-L. 2009. Improved genetic algorithm for resource-constrained scheduling of large projects. Canadian Journal of Civil Engineering, 36(6): 1016-1027. doi:10.1139/L09-049.

28 Koulinas, G.K., and Anagnostopoulos, K.P. 2012. Construction Resource Allocation and Leveling Using a 
Threshold Accepting-Based Hyperheuristic Algorithm. Journal of Construction Engineering and Management, 138(7): 854-863. doi:10.1061/(ASCE)CO.1943-7862.0000492.

Koulinas, G.K., and Anagnostopoulos, K.P. 2013. A new tabu search-based hyper-heuristic algorithm for solving construction leveling problems with limited resource availabilities. Automation in Construction, 31: 169-175. Elsevier. doi:10.1016/J.AUTCON.2012.11.002.

Leu, S.-S., and Hung, T.-H. 2002. An optimal construction resource leveling scheduling simulation model. Canadian Journal of Civil Engineering, 29(2): 267-275. NRC Research Press Ottawa, Canada . doi:10.1139/102-007.

Leu, S.S., Yang, C.H., and Huang, J.C. 2000. Resource leveling in construction by genetic algorithm-based optimization and its decision support system application. Automation in construction, 10(1): 27-41.

Menesi, W., and Hegazy, T. 2015. Multimode Resource-Constrained Scheduling and Leveling for Practical-Size Projects. Journal of Management in Engineering, 31(6): 04014092. doi:10.1061/(ASCE)ME.1943-

Moradi, M., Delavar, M.R., and Moshiri, B. 2017. A GIS-based multi-criteria analysis model for earthquake vulnerability assessment using Choquet integral and game theory. Natural Hazards, 87(3): 1377-1398.

(1)

$$
\text { Springer Netherlands. doi:10.1007/s11069-017-2822-6. }
$$

Najibi, N., and Devineni, N. 2018. Recent trends in the frequency and duration of global floods. Earth System Dynamics, 9(2): 757-783. doi:10.5194/esd-9-757-2018.

Padilla, E.M., and Carr, R.I. 1991. Resource Strategies for Dynamic Project Management. Journal of Construction Engineering and Management, 117(2): 279-293. doi:10.1061/(ASCE)0733-9364(1991)117:2(279).

Peteghem, V. Van, and Vanhoucke, M. 2010. A genetic algorithm for the preemptive and non-preemptive multimode resource-constrained project scheduling problem. European Journal of Operational Research, 201(2): 409-418. North-Holland. doi:10.1016/J.EJOR.2009.03.034.

Piryonesi, S.M., and Tavakolan, M. 2017. A mathematical programming model for solving cost-safety optimization (CSO) problems in the maintenance of structures. KSCE Journal of Civil Engineering, 21(6): 4.

28 Ponz-Tienda, J.L., Yepes, V., Pellicer, E., and Moreno-Flores, J. 2013. The Resource Leveling Problem with 
multiple resources using an adaptive genetic algorithm. Automation in Construction, 29(1): 161-172. Elsevier B.V. doi:10.1016/j.autcon.2012.10.003.

3 Rieck, J., Zimmermann, J., and Gather, T. 2012. Mixed-integer linear programming for resource leveling problems.

4 European Journal of Operational Research, 221(1): 27-37. North-Holland. doi:10.1016/J.EJOR.2012.03.003.

5 Son, J., and Skibniewski, M.J. 1999. Multiheuristic Approach for Resource Leveling Problem in Construction

6 Engineering: Hybrid Approach. Journal of Construction Engineering and Management, 125(1): $23-31$.

7 doi:10.1061/(ASCE)0733-9364(1999)125:1(23).

8 Toklu, Y.C. 2002. Application of genetic algorithms to construction scheduling with or without resource constraints.

9 Canadian Journal of Civil Engineering, 29(3): 421-429. NRC Research Press Ottawa, Canada .

10 doi:10.1139/102-034.

11 Wiest, J.D., and Levy, F.K. 1977. A Management Guide to PERT/CPM. Prentice Hall, , Englewood Cliffs, NJ.

12 Zahraie, B., and Tavakolan, M. 2009. Stochastic Time-Cost-Resource Utilization Optimization Using

13 Nondominated Sorting Genetic Algorithm and Discrete Fuzzy Sets. Journal of Construction Engineering and

14 Management, 135(11): 1162-1171. doi:10.1061/(ASCE)CO.1943-7862.0000092.

15

16

17 